\title{
Minimum Transversals in Posi-modular Systems *
}

\author{
Mariko Sakashita $^{\dagger} \quad$ Kazuhisa Makino ${ }^{\ddagger}$ Hiroshi Nagamochi ${ }^{\S}$ \\ Satoru Fujishige
}

\begin{abstract}
Given a system $(V, f, d)$ on a finite set $V$ consisting of two set functions $f: 2^{V} \rightarrow \mathbb{R}$ and $d: 2^{V} \rightarrow \mathbb{R}$, we consider the problem of finding a set $R \subseteq V$ of minimum cardinality such that $f(X) \geq d(X)$ for all $X \subseteq V-R$, where the problem can be regarded as a natural generalization of the source location problems and the external network problems in (undirected) graphs and hypergraphs. We give a structural characterization of minimal deficient sets of $(V, f, d)$ under certain conditions. We show that all such sets form a tree hypergraph if $f$ is posi-modular and $d$ is modulotone (i.e., each nonempty subset $X$ of $V$ has an element $v \in X$ such that $d(Y) \geq d(X)$ for all subsets $Y$ of $X$ that contain $v$ ), and that conversely any tree hypergraph can be represented by minimal deficient sets of $(V, f, d)$ for a posi-modular function $f$ and a modulotone function $d$. By using this characterization, we present a polynomial-time algorithm if, in addition, $f$ is submodular and $d$ is given by either $d(X)=\max \{p(v) \mid v \in X\}$ for a function $p: V \rightarrow \mathbb{R}_{+}$or $d(X)=\max \{r(v, w) \mid v \in X, w \in V-X\}$ for a function $r: V^{2} \rightarrow \mathbb{R}_{+}$. Our result provides first polynomial-time algorithms for the source location problem in hypergraphs and the external network problems in graphs and hypergraphs. We also show that the problem is intractable, even if $f$ is submodular and $d \equiv \mathbf{0}$.
\end{abstract}

\section{Introduction}

Given a system $(V, f, d)$ on a finite set $V$ consisting of two set functions $f: 2^{V} \rightarrow \mathbb{R}$ and $d: 2^{V} \rightarrow \mathbb{R}$ with $f(\emptyset) \geq d(\emptyset)$, we consider the problem

${ }^{*}$ A preliminary version is to appear in The 14th Annual European Symposium on Algorithms (ESA2006) 11-13 September 2006.

${ }^{\dagger}$ Graduate School of Informatics, Kyoto University, Kyoto, 606-8501, Japan. sakasita@amp.i.kyoto-u.ac.jp

${ }^{\ddagger}$ Graduate School of Information Science and Technology, University of Tokyo, Tokyo, 113-8656, Japan. makino@mist.i.u-tokyo.ac.jp

${ }^{\S}$ Graduate School of Informatics, Kyoto University, Kyoto, 606-8501, Japan. nag@amp.i.kyoto-u.ac.jp

${ }^{\top}$ Research Institute for Mathematical Sciences, Kyoto University, Kyoto, 606-8502, Japan. fujishig@kurims.kyoto-u.ac.jp 
of finding a set $R \subseteq V$ of minimum cardinality such that $f(X) \geq d(X)$ for all $X \subseteq V-R$. The problem can be regarded as a natural generalization of the source location problems and the external network problems with edge-connectivity requirements in (undirected) graphs and hypergraphs [1, $7,8,14]$; we will discuss these problems in Section 6 . We give an interesting structural characterization of minimal deficient sets of $(V, f, d)$, i.e., minimal sets $X \subseteq V$ such that $f(X)<d(X)$, under certain conditions. We show that all such sets form a tree hypergraph if $f$ is posi-modular and $d$ is modulotone (i.e., each nonempty subset $X$ of $V$ has an element $v \in X$ such that $d(Y) \geq d(X)$ for all subsets $Y$ of $X$ containing $v$ ), and that conversely any tree hypergraph can be represented by minimal deficient sets of $(V, f, d)$ for a posi-modular function $f$ and a modulotone function $d$. By using this characterization, we present a polynomial-time algorithm when $f$ is in addition submodular and $d$ is given by either $d(X)=\max \{p(v) \mid v \in X\}$ for a function $p: V \rightarrow \mathbb{R}_{+}$or $d(X)=\max \{r(v, w) \mid v \in X, w \in V-X\}$ for a function $r: V^{2} \rightarrow \mathbb{R}_{+}$.

As applications of our algorithm, we present first polynomial-time algorithms for the following problems:

1. The source location problem in hypergraphs with edge-connectivity requirements.

2. The external network problems in graphs and hypergraphs with edgeconnectivity requirements.

We also show that the problem is intractable even if $f$ is submodular and $d \equiv \mathbf{0}$. Namely, we show that the problem is NP-hard if a submodular function $f$ is given as a functional form, and it requires at least $2^{\frac{n}{2}}$ time in the worst case, if $f$ is given implicitly by an oracle, where $n=|V|$.

Our approach partly follows the idea by Bárász et al. [2] that is used to construct a polynomial-time algorithm for the source location problem with a uniform demand function in directed networks. They introduced a new concept of solid sets for cut functions of directed graphs, proved that solid sets form a tree hypergraph, and gave an efficient algorithm for computing the underlying tree of the tree hypergraph by introducing a technique to reduce the size of solid sets required to obtain the tree. However, their proof is based on the properties of cut functions $f$ of directed graphs (which cannot be generalized to submodular functions, as will be observed in Proposition 8) and the uniformness of demand functions (which cannot also be generalized to modulotonicity), while our proof is based on the posi-modularity of $f$ and modulotonicity of demand functions.

The rest of this paper is organized as follows. In Section 2 we formulate our problem, introduce some known results, and present our structural result on the minimal deficient sets. Section 3 shows the intractability of the problem, even if $f$ is submodular. Section 4 reveals the structural properties 
of posi-modular systems and gives a proof of our new hypertree characterization (Theorem 4). Section 5 describes a polynomial-time algorithm if $f$ is submodular and posi-modular. Section 6 addresses applications of our problem. Finally, Section 7 concludes the paper.

\section{Preliminaries}

\section{$2.1 \quad$ Hypergraphs}

Let $V$ be a finite set. A family $\mathcal{E} \subseteq 2^{V}$ is called a Sperner family if for arbitrary two distinct sets $E, E^{\prime} \in \mathcal{E}$, neither $E \subseteq E^{\prime}$ nor $E^{\prime} \subseteq E$ holds. For a family $\mathcal{E} \subseteq 2^{V}$, the hypergraph $(V, \mathcal{E})$ is simply written as $\mathcal{E}$. For a hypergraph $\mathcal{E}$, a subset $R \subseteq V$ is called a transversal (or hitting set) of $\mathcal{E}$ if $R \cap E \neq \emptyset$ for all $E \in \mathcal{E}$. Let $\tau(\mathcal{E})$ denote the transversal number of $\mathcal{E}$, i.e.,

$$
\tau(\mathcal{E})=\min \{|R| \mid R \text { is a transversal of } \mathcal{E}\} .
$$

A subfamily $\mathcal{E}^{\prime} \subseteq \mathcal{E}$ is called a matching of $\mathcal{E}$ if $E \cap E^{\prime}=\emptyset$ for arbitrary two distinct sets $E, E^{\prime} \in \mathcal{E}^{\prime}$, and let $\nu(\mathcal{E})$ denote the matching number of $\mathcal{E}$, i.e.,

$$
\nu(\mathcal{E})=\max \left\{\left|\mathcal{E}^{\prime}\right| \mid \mathcal{E}^{\prime} \text { is a matching of } \mathcal{E}\right\} .
$$

A hypergraph $\mathcal{E}$ is called a tree hypergraph (or hypertree) if there exists a tree $T$ with a vertex set $V$ such that each hyperedge $E \in \mathcal{E}$ induces a subtree of $T$. Such a tree $T$ is called a basic tree for the hypergraph $\mathcal{E}$. For a tree hypergraph, the following result is known.

Theorem 1 (e.g., [3]) Let $\mathcal{E}$ be a tree hypergraph. Then $\mathcal{E}$ satisfies the König property, i.e., $\tau(\mathcal{E})=\nu(\mathcal{E})$.

We review two characterizations of tree hypergraphs. A hypergraph $\mathcal{E}$ is said to have the Helly property if every subfamily of pairwise intersecting hyperedges has a nonempty intersection. The line (or intersecting) graph $L(\mathcal{E})$ of a hypergraph $\mathcal{E}$ is a graph in which the vertices correspond to the hyperedges, two of them being adjacent if the corresponding hyperedges have a nonempty intersection. An undirected graph is called chordal if every cycle of length at least 4 has a chord.

Theorem 2 (e.g., [11]) A family $\mathcal{E} \subseteq 2^{V}$ is a tree hypergraph if and only if $\mathcal{E}$ has the Helly property and its line graph $L(\mathcal{E})$ is chordal.

Another characterization is known as follows. Define a weight function $c(u, v)$ on the edge set of the complete graph on $V$ as follows. For every pair $\{u, v\}$ of elements in $V$, let $c(u, v)$ be the number of hyperedges containing both $u$ and $v$. 
Theorem 3 (Bárász et al.[2]) A family $\mathcal{E}$ is a tree hypergraph if and only if a spanning tree of maximum c-weight has weight $\sum_{E \in \mathcal{E}}(|E|-1)$. Furthermore, such a spanning tree is a basic tree for $\mathcal{E}$.

It follows from Theorem 3 that any maximum spanning tree algorithm can be used to compute a basic tree for a tree hypergraph.

\subsection{Transversals over set functions}

In this paper, we consider a system $(V, f, d)$ on a finite set $V$ consisting of two set functions $f: 2^{V} \rightarrow \mathbb{R}$ and $d: 2^{V} \rightarrow \mathbb{R}$ with $f(\emptyset) \geq d(\emptyset)$, and introduce the following problem:

$$
\begin{array}{ll}
\text { Minimize } & |R| \\
\text { subject to } & f(X) \geq d(X) \text { for all } X \subseteq V-R \\
& R \subseteq V .
\end{array}
$$

Here $f(\emptyset) \geq d(\emptyset)$ is necessary for the problem to have a feasible solution. A vertex subset $X \subseteq V$ is called deficient if $f(X)<d(X)$. A deficient set $X$ is called minimal if no proper subset of $X$ is deficient. Let $\mathcal{W}(f, d)$ denote the family of all minimal deficient sets of $(V, f, d)$. Then the constraint in problem (1) is equivalent to

$$
R \cap X \neq \emptyset \text { for all } X \in \mathcal{W}(f, d) \text {. }
$$

Therefore, it follows that the problem we consider is to compute a minimum transversal $R$ of $\mathcal{W}(f, d)$. We remark that $\mathcal{W}(f, d)$ is not given explicitly and $|\mathcal{W}(f, d)|$ may be exponential in $n=|V|$.

A set function $f: 2^{V} \rightarrow \mathbb{R}$ is called submodular if

$$
f(X)+f(Y) \geq f(X \cup Y)+f(X \cap Y)
$$

for arbitrary two subsets $X, Y$ of $V$, and posi-modular if

$$
f(X)+f(Y) \geq f(X-Y)+f(Y-X)
$$

for arbitrary two subsets $X, Y$ of $V$. We call a function $d: 2^{V} \rightarrow \mathbb{R}$ modulotone if each nonempty $X \subseteq V$ has an element $v \in X$ such that $d(Y) \geq d(X)$ for all $Y \subseteq X$ containing $v$.

One of the main contributions of this paper is to derive the following new characterization of tree hypergraphs in terms of set functions.

Theorem 4 A Sperner family $\mathcal{E} \subseteq 2^{V}$ is a tree hypergraph if and only if $\mathcal{E}=\mathcal{W}(f, d)$ holds for a posi-modular function $f: 2^{V} \rightarrow \mathbb{R}$ and a modulotone function $d: 2^{V} \rightarrow \mathbb{R}$. 


\section{$3 \quad$ Submodular Systems}

This section shows that problem (1) with a submodular function $f$ is intractable in general and $\mathcal{W}(f, d)$ may not be a tree hypergraph. We first show that every Sperner hypergraph $\mathcal{E}$ can be represented by $\mathcal{W}(f, d)$ of a submodular function $f$ and a constant function $d$.

Lemma 5 For a Sperner hypergraph $\mathcal{E} \subseteq 2^{V}$, let $d, f: 2^{V} \rightarrow \mathbb{R}$ be functions defined by

$$
\begin{aligned}
& d(X)=0 \text { for all } X \subseteq V, \\
& f(X)=-|\mathcal{E}(X)| \text { for all } X \subseteq V,
\end{aligned}
$$

where $\mathcal{E}(X)=\{E \in \mathcal{E} \mid E \subseteq X\}$. Then $f$ is submodular and it holds that

$$
\mathcal{E}=\mathcal{W}(f, d) .
$$

Proof. By definition, a subset $X$ of $V$ is deficient if and only if $X$ contains at least one hyperedge $E$ in $\mathcal{E}$. Since $\mathcal{E}$ is Sperner, it follows that the family $\mathcal{W}(f, d)$ of all minimal deficient sets is given by $\mathcal{W}(f, d)=\mathcal{E}$.

We next show that $f$ is submodular, which completes the proof. It is easy to see that $|\mathcal{E}(X)|+|\mathcal{E}(Y)| \leq|\mathcal{E}(X \cap Y)|+|\mathcal{E}(X \cup Y)|$ for arbitrary $X$, $Y \subseteq V$, since $\mathcal{E}(X), \mathcal{E}(Y) \subseteq \mathcal{E}(X \cup Y)$ and $\mathcal{E}(X) \cap \mathcal{E}(Y)=\mathcal{E}(X \cap Y)$. Then we have

$$
\begin{aligned}
f(X)+f(Y) & =-|\mathcal{E}(X)|-|\mathcal{E}(X)| \\
& \geq-|\mathcal{E}(X \cap Y)|-|\mathcal{E}(X \cup Y)| \\
& =f(X \cap Y)+f(X \cup Y),
\end{aligned}
$$

i.e., $f$ is submodular.

Since it is NP-hard to compute a minimum transversal of a general Sperner hypergraph, Lemma 5 implies the following negative result.

Theorem 6 Let $d$ be a function defined by $d \equiv \mathbf{0}$. For a given Sperner hypergraph $\mathcal{E} \subseteq 2^{V}$, let $f$ be a submodular function given by (6). Then it is $\mathrm{NP}$-hard to compute a minimum transversal of $\mathcal{W}(f, d)$.

We also have the following negative result if $f$ is given by an oracle, i.e., we can invoke an oracle for the evaluation of $f(X)$ for any $X \subseteq V$ and use the function value $f(X)$.

Theorem 7 Let $f$ be a submodular function given by an oracle. Then problem (1) requires at least $2^{\frac{n}{2}}$ calls to the oracle in the worst case, where $n=|V|$. 
Proof. Let us assume a contrary that there exists an algorithm (called A) that solves problem (1) by calling the oracle at most $2^{\frac{n}{2}}-1$ times.

Let $V=\{1, \ldots, n\}$, where $n$ is even. Let $\mathcal{E}$ be a Sperner hypergraph on $V$ defined by $\mathcal{E}=\mathcal{E}_{1} \cup \mathcal{E}_{2}$, where

$$
\begin{aligned}
& \mathcal{E}_{1}=\left\{\{2 j-1,2 j\} \mid j=1, \ldots, \frac{n}{2}\right\} \\
& \mathcal{E}_{2}=\left\{E \subseteq V|| E \cap\{2 j-1,2 j\} \mid=1 \text { for all } j=1, \ldots, \frac{n}{2}\right\} .
\end{aligned}
$$

We then apply algorithm A to problem (1) in which $f$ is given by (6) and $d$ given by $d \equiv \mathbf{0}$. Since $\left|\mathcal{E}_{2}\right|=2^{\frac{n}{2}}$, there exists a hyperedge $E^{*}$ in $\mathcal{E}_{2}$ such that $f\left(E^{*}\right)$ is not evaluated by the oracle. Take such a hyperedge $E^{*}$ arbitrarily. Let $f^{\prime}$ be a function on $V$ defined by

$$
f^{\prime}(S)= \begin{cases}f(S) & \text { if } S \neq E^{*} \\ f(S)+1(=0) & \text { otherwise }\end{cases}
$$

Then it is not difficult to see that $f^{\prime}$ is submodular. Note that $T$ is a minimum transversal of system $(V, f, d)$ if and only if it satisfies $|T|=\frac{n}{2}+1$ and $T \cap E \neq \emptyset$ for all $E \in \mathcal{E}_{1}$, while $T^{\prime}$ is a minimum transversal of system $\left(V, f^{\prime}, d\right)$ if and only if $T^{\prime}=V-E^{*}$, which implies $\left|T^{\prime}\right|=\frac{n}{2}(\neq|T|)$. Since $f(S)=f^{\prime}(S)$ for all $S$ with $S \neq E^{*}$, and algorithm A does not evaluate $f\left(E^{*}\right)$, this is a contradiction.

Lemma 5 also implies that $\mathcal{W}(f, d)$ is not a tree hypergraph even if $f$ is submodular, which contrasts to the result on posi-modular functions $f$.

Proposition 8 The family $\mathcal{W}(f, d)$ is not always a tree hypergraph, even if $f$ is a submodular function and $d$ is given by $d \equiv \mathbf{0}$.

Proof. We give an instance such that $\mathcal{W}(f, d)$ is not a tree hypergraph. Let $\mathcal{E}$ be a hypergraph given by

$$
V=\left\{v_{1}, v_{2}, v_{3}, v_{4}\right\} \text { and } \mathcal{E}=\left\{\left\{v_{1}, v_{2}\right\},\left\{v_{2}, v_{3}\right\},\left\{v_{3}, v_{4}\right\},\left\{v_{4}, v_{1}\right\}\right\},
$$

i.e., $\mathcal{E}$ is an undirected graph that forms a simple cycle of length 4 . From Lemma 5, we have $\mathcal{E}=\mathcal{W}(f, d)$ for a submodular function $f$ given by (6) and $d$ given by $d \equiv \mathbf{0}$. The line graph $L$ of $\mathcal{W}(f, d)$ has a cycle of length 4 and this cycle has no chord. That is, $L$ is not chordal and hence by Theorem $2 \mathcal{W}(f, d)$ is not a tree hypergraph.

\section{Posi-modular Systems}

This section discusses problem (1) for posi-modular functions $f$. We first prove the sufficiency part of Theorem 4 , where the necessity will be shown in Section 4.2. 


\subsection{Structure of posi-modular functions}

Let $V$ be a finite set and $f: 2^{V} \rightarrow \mathbb{R}$ be a posi-modular function.

\subsubsection{Basic properties of posi-modular functions:}

This section gives two properties of posi-modular functions.

Lemma 9 Let $X_{0}, X_{1}, \ldots, X_{h-1}, X_{h}\left(=X_{0}\right)(h \geq 3)$ be subsets of $V$ such that $X_{i} \cap X_{j} \neq \emptyset$ if and only if $i$ and $j$ are consecutive integers. For each $i=0, \ldots, h-1$, let $Y_{i}=X_{i} \cap X_{i+1}$. Then any posi-modular function $f$ satisfies

$$
\sum_{i=0}^{h-1} f\left(X_{i}\right) \geq \sum_{i=0}^{h-1} f\left(Y_{i}\right) .
$$

Proof. We have

$$
\begin{aligned}
2 \sum_{i=0}^{h-1} f\left(X_{i}\right) & =\sum_{i=0}^{h-1}\left\{f\left(X_{i}\right)+f\left(X_{i+1}\right)\right\} \\
& \geq \sum_{i=0}^{h-1}\left\{f\left(X_{i}-X_{i+1}\right)+f\left(X_{i+1}-X_{i}\right)\right\} \quad \text { (by posi-modularity of } f \text { ) } \\
& =\sum_{i=0}^{h-1}\left\{f\left(X_{i+1}-X_{i}\right)+f\left(X_{i+1}-X_{i+2}\right)\right\} \\
& \geq \sum_{i=0}^{h-1}\left\{f\left(X_{i+1} \cap X_{i}\right)+f\left(X_{i+1} \cap X_{i+2}\right)\right\} \quad \text { (by posi-modularity of } f \text { ) } \\
& =\sum_{i=0}^{h-1}\left\{f\left(Y_{i}\right)+f\left(Y_{i+1}\right)\right\} \quad=2 \sum_{i=0}^{h-1} f\left(Y_{i}\right),
\end{aligned}
$$

where $X_{h+1}=X_{1}$, and the second inequality follows from the fact that $\left(X_{i+1}-X_{i}\right)-\left(X_{i+1}-X_{i+2}\right)=\left(X_{i+1}-X_{i}\right) \cap X_{i+2}$ and $X_{i} \cap X_{i+2}=\emptyset$.

Let $\mathcal{X}=\left\{X_{1}, \ldots, X_{h}\right\} \subseteq 2^{V}$ and $I=\{1, \ldots, h\}$. For each subset $J$ of $I$, let

$$
Z_{J}=\bigcap_{j \in J} X_{j}
$$

Lemma 10 If $\mathcal{X}$ is pairwise intersecting, i.e., $X_{i} \cap X_{j} \neq \emptyset$ for all $i, j \in I$, then for any posi-modular function $f$ on $2^{V}$, we have

$$
\sum_{i=1}^{h} f\left(X_{i}\right) \geq \sum_{i=1}^{h} f\left(Z_{I \backslash\{i\}}-Z_{I}\right) .
$$


Proof. We proceed by induction on the size $h$ of $\mathcal{X}$. For $h=2$, we can prove it directly from the posi-modularity of $f$ since $Z_{I \backslash\{1\}}-Z_{I}=X_{2}-X_{1}$ and $Z_{I \backslash\{2\}}-Z_{I}=X_{1}-X_{2}$. Let $\ell$ be an integer with $\ell \geq 2$. Assuming that the statement in the lemma is true for $h=\ell$, we consider the case when $h=\ell+1$. By the inductive hypothesis, we have

$$
\begin{aligned}
(h-1) & \sum_{i \in I} f\left(X_{i}\right) \\
= & \sum_{i \in I} \sum_{j \in I \backslash\{i\}} f\left(X_{j}\right) \\
\geq & \sum_{i \in I} \sum_{j \in I \backslash\{i\}} f\left(Z_{I \backslash\{i, j\}}-Z_{I \backslash\{i\}}\right) \quad \text { (by inductive hypothesis) } \\
= & \sum_{i, j \in I: i<j}\left\{f\left(Z_{I \backslash\{i, j\}}-Z_{I \backslash\{i\}}\right)+f\left(Z_{I \backslash\{i, j\}}-Z_{I \backslash\{j\}}\right)\right\} \\
\geq & \sum_{i, j \in I: i<j}\left\{f\left(Z_{I \backslash\{i\}}-Z_{I}\right)+f\left(Z_{I \backslash\{j\}}-Z_{I}\right)\right\} \quad \text { (by posi-modularity of } f \text { ) } \\
= & (h-1) \sum_{i \in I} f\left(Z_{I \backslash\{i\}}-Z_{I}\right),
\end{aligned}
$$

where we note that in the second inequality $\left(Z_{I \backslash\{i, j\}}-Z_{I \backslash\{i\}}\right)-\left(Z_{I \backslash\{i, j\}}-\right.$ $\left.Z_{I \backslash\{j\}}\right)=\left(Z_{I \backslash\{i, j\}}-Z_{I \backslash\{i\}}\right) \cap Z_{I \backslash\{j\}}=Z_{I \backslash\{j\}}-Z_{I}$.

\subsubsection{Solid sets}

For an element $v \in V$, we call a nonempty subset $X$ of $V$-solid (with respect to $f$ ) if $v \in X$ and $f(X)<f(Y)$ for all nonempty proper subsets $Y$ of $X$ containing $v$. For each $v \in V$, we denote by $\mathcal{S}_{v}$ the family of all $v$-solid sets. Let $\mathcal{S}(f)=\bigcup_{v \in V} \mathcal{S}_{v}$. We prove that $\mathcal{S}(f)$ is a tree hypergraph if $f$ is posi-modular. For each subset $X \subseteq V$, let $A_{X}=\left\{v \in X \mid X \in \mathcal{S}_{v}\right\}$.

Lemma 11 Let $X$ and $Y$ be sets in $\mathcal{S}(f)$ such that $X \cap Y \neq \emptyset$. Then $A_{X}$ or $A_{Y}$ is included in $X \cap Y$, if $f$ is a posi-modular function.

Proof. We suppose that $X-Y$ and $Y-X$ are both nonempty, since the lemma clearly holds if $X \subseteq Y$ or $Y \subseteq X$. By the posi-modularity, we have

$$
f(X) \geq f(X-Y) \quad \text { or } \quad f(Y) \geq f(Y-X) .
$$

By symmetry, we assume without loss of generality that $f(X) \geq f(X-Y)$. Then $X$ cannot be $v$-solid for any $v \in X-Y$ since $X-Y$ is a nonempty proper subset of $X$. Therefore all elements $v \in X$ such that $X \in \mathcal{S}_{v}$ belong to $X \cap Y$, i.e., $A_{X} \subseteq X \cap Y$. 
Lemma 12 The line graph $L$ of $\mathcal{S}(f)$ is chordal, if $f$ is a posi-modular function.

Proof. Assuming that $L$ is not chordal, we derive a contradiction. Let $X_{0}$, $X_{1}, \ldots, X_{h-1}, X_{h}\left(=X_{0}\right)$ be a chordless cycle in $\mathcal{S}(f)$ of length at least 4 . For each $i=0, \ldots, h-1$, let $Y_{i}=X_{i} \cap X_{i+1}$. Then we have $Y_{i} \neq \emptyset$ for all $i=0, \ldots, h-1$ and $Y_{i} \cap Y_{j}=\emptyset$ for all $i$ and $j$ with $i \neq j$. It follows from Lemma 11 that

$$
A_{X_{0}} \subseteq Y_{0} \quad \text { or } \quad A_{X_{1}} \subseteq Y_{0}
$$

By symmetry, we assume without loss of generality that $A_{X_{1}} \subseteq Y_{0}$. Then by applying Lemma 11 to $X_{1}$ and $X_{2}, A_{X_{2}} \subseteq Y_{1}$ holds, since $Y_{0} \cap Y_{1}=\emptyset$. By repeating this argument, we have

$$
A_{X_{i+1}} \subseteq Y_{i} \quad \text { for } i=0,1, \ldots, h-1 .
$$

From this,

$$
f\left(Y_{i}\right)>f\left(X_{i+1}\right) \quad \text { for } i=0,1, \ldots, h-1
$$

since $Y_{i}$ is a proper subset of $X_{i+1}$ containing some $v$ with $X_{i+1} \in \mathcal{S}_{v}$. Therefore we have

$$
\sum_{i=0}^{h-1} f\left(Y_{i}\right)>\sum_{i=0}^{h-1} f\left(X_{i}\right),
$$

which contradicts Lemma 9.

Let us next show the Helly property of $\mathcal{S}(f)$ by extending Lemma 11.

Lemma 13 Let $f$ be a posi-modular function, and let $\mathcal{X}=\left\{X_{i} \mid i \in I=\right.$ $\{1, \ldots, h\}\}$ be a pairwise intersecting subfamily of $\mathcal{S}(f)$. Then it has a set $X_{i}$ such that $A_{X_{i}} \subseteq Z_{I}$, where $Z_{I}$ is given by (7).

Proof. We proceed by induction on the size $h$ of $\mathcal{X}$. We first note that the statement in the lemma holds from Lemma 11 when $h=2$.

Let $\ell$ be an integer with $\ell \geq 2$. Assuming that the statement in the lemma is true for $h=\ell$, we consider the case when $h=\ell+1$. Let us assume that $A_{X_{i}} \nsubseteq Z_{I}$ for all $i \in I$, and we derive a contradiction. By the inductive hypothesis and the assumption, for each $j \in I$ there exists an $i(j) \in I \backslash\{j\}$ such that

$$
A_{X_{i(j)}} \subseteq Z_{I \backslash\{j\}} \quad \text { and } \quad A_{X_{i(j)}} \cap\left(Z_{I \backslash\{j\}}-Z_{I}\right) \neq \emptyset .
$$

We also have

$$
f\left(X_{i(j)}\right)<f\left(Z_{I \backslash\{j\}}-Z_{I}\right)
$$


since $Z_{I \backslash\{j\}}-Z_{I}$ is a proper subset of $X_{i(j)}$ containing some $v$ with $X_{i(j)} \in \mathcal{S}_{v}$. Note that $i(j) \neq i\left(j^{\prime}\right)$ for $j \neq j^{\prime}$, since otherwise we would have

$$
A_{X_{i(j)}}=A_{X_{i\left(j^{\prime}\right)}} \subseteq Z_{I \backslash\{j\}} \cap Z_{I \backslash\left\{j^{\prime}\right\}}=Z_{I},
$$

which contradicts the assumption. This implies that $I=\{i(j) \mid j \in I\}$. Therefore we have

$$
\sum_{i=1}^{h} f\left(X_{i}\right)<\sum_{i=1}^{h} f\left(Z_{I \backslash\{i\}}-Z_{I}\right),
$$

which contradicts Lemma 10.

Lemma 13 directly implies the following.

Corollary 14 If $f$ is posi-modular, then $\mathcal{S}(f)$ has the Helly property.

Lemma 12 and Corollary 14 imply the following theorem.

Theorem 15 If $f$ is posi-modular, then $\mathcal{S}(f)$ is a tree hypergraph.

We are ready to prove the sufficiency part of Theorem 4 .

Lemma 16 If $f$ is a posi-modular function and $d$ is a modulotone function, then $\mathcal{W}(f, d) \subseteq \mathcal{S}(f)$.

Proof. Let $X$ be a member of $\mathcal{W}(f, d)$. Then $f(X)<d(X)$ and $f(Y) \geq d(Y)$ for all nonempty proper subsets $Y$ of $X$. From the assumption on $d$, there is an element $v \in X$ such that $d(Y) \geq d(X)$ for all $Y \subseteq X$ containing $v$. Therefore we have $f(Y) \geq d(Y) \geq d(X)>f(X)$ for all proper subsets $Y$ of $X$ containing $v$. That is, $X$ is $v$-solid and hence $X \in \mathcal{S}(f)$ holds.

Theorem 15 together with Lemma 16 implies the sufficiency part of Theorem 4 .

\subsection{Necessity Part of Theorem 4}

Let us show the necessity part of Theorem 4 .

For a hypergraph $\mathcal{E} \subseteq 2^{V}$, let $w: \mathcal{E} \rightarrow \mathbb{R}_{+}$be a nonnegative weight function on $\mathcal{E}$. Let us define $f, d: 2^{V} \rightarrow \mathbb{R}$ by

$$
\begin{aligned}
f(X) & =\sum\{w(E) \mid E \in \mathcal{E}, E \cap X \neq \emptyset, E-X \neq \emptyset\} \\
d(X) & =\max _{v \in X} d(v),
\end{aligned}
$$

where $d(\emptyset)=0$ and

$$
d(v)=\sum\{w(E) \mid v \in E \in \mathcal{E}\} .
$$

Note that $f$ is a cut function of the hypergraph. This implies that $f$ is symmetric submodular, and hence it is posi-modular. It is clear that $d$ : $2^{V} \rightarrow \mathbb{R}_{+}$is modulotone. 
Lemma 17 For any weight function $w: \mathcal{E} \rightarrow \mathbb{R}_{+}, \mathcal{W}(f, d)$ defined by $f$ and $d$ by (8) has a property that each set $X \in \mathcal{W}(f, d)$ contains a set $E \in \mathcal{E}$.

Proof. Let $X \in \mathcal{W}(f, d)$. Since $f(\emptyset)=d(\emptyset)=0, X \neq \emptyset$ holds. Let $v \in X$ be an element such that $d(v)=d(X)$. Then we have $d(X)=d(v) \leq \sum\{w(E) \mid$ $E \in \mathcal{E}, E \cap X \neq \emptyset\}$. Assume that $E \nsubseteq X$ holds for all $E \in \mathcal{E}$. Then we have

$$
f(X)=\sum\{w(E) \mid E \in \mathcal{E}, E \cap X \neq \emptyset\} \geq d(X),
$$

which implies that $X$ is not deficient. Therefore, $X \in \mathcal{W}(f, d)$ contains some set $E \in \mathcal{E}$.

From Lemma 17, we can see that $E \in \mathcal{E}$ is minimal deficient if it is deficient.

Let $\mathcal{E}$ be a tree Sperner hypergraph with $|\mathcal{E}|=m$. Then by Theorem 2, $\mathcal{E}$ admits an ordering of edges $\sigma=\left[E_{1}, E_{2}, \ldots, E_{m}\right]$ such that

$$
\bigcap\left\{E_{j} \in \mathcal{E} \mid E_{j} \cap E_{i} \neq \emptyset, j>i\right\} \neq \emptyset, i=1, \ldots, m,
$$

if $\left\{E_{j} \in \mathcal{E} \mid E_{j} \cap E_{i} \neq \emptyset, j>i\right\} \neq \emptyset$. Here we note that this ordering corresponds to a perfect elimination ordering of the line graph $L(\mathcal{E})$ [11]. Let

$$
\begin{aligned}
\mathcal{E}_{i}^{\downarrow} & =\left\{E_{j} \in \mathcal{E} \mid E_{j} \cap E_{i} \neq \emptyset, j<i\right\}, \\
\mathcal{E}_{i}^{\uparrow} & =\left\{E_{j} \in \mathcal{E} \mid E_{j} \cap E_{i} \neq \emptyset, j>i\right\}, \text { and } \\
\mathcal{E}_{i} & =\mathcal{E}_{i}^{\downarrow} \cup \mathcal{E}_{i}^{\uparrow} .
\end{aligned}
$$

Then (9) is equivalent to the following claim.

Claim 18 For all $i=1, \ldots, m, \mathcal{E}_{i}^{\uparrow} \neq \emptyset$ implies that $\bigcap\left\{E \mid E \in \mathcal{E}_{i}^{\uparrow}\right\} \neq \emptyset$.

We define a weight function $w: \mathcal{E} \rightarrow \mathbb{R}_{+}$by

$$
w\left(E_{i}\right)=\sum\left\{w(E) \mid E \in \mathcal{E}_{i}^{\downarrow}\right\}+1 \quad \text { for } i=1, \ldots m .
$$

For this weight function $w$, we have the following lemma.

Lemma 19 Each $E_{i} \in \mathcal{E}$ is deficient.

Proof. For a hyperedge $E_{i} \in \mathcal{E}$, we separately consider two cases: (i) $\mathcal{E}_{i}^{\uparrow}=\emptyset$ and (ii) $\mathcal{E}_{i}^{\uparrow} \neq \emptyset$.

(i) If $\mathcal{E}_{i}^{\uparrow}=\emptyset$, then from the Sperner property of $\mathcal{E}$, we have

$$
f\left(E_{i}\right)=\sum\left\{w(E) \mid E \in \mathcal{E}_{i}^{\downarrow}\right\}<w\left(E_{i}\right) \leq d\left(E_{i}\right) .
$$


(ii) If $\mathcal{E}_{i}^{\uparrow} \neq \emptyset$, then from Claim 18 and the Sperner property of $\mathcal{E}$, we have

$$
\begin{aligned}
d\left(E_{i}\right) & \geq w\left(E_{i}\right)+\sum\left\{w(E) \mid E \in \mathcal{E}_{i}^{\uparrow}\right\} \\
& =\sum\left\{w(E) \mid E \in \mathcal{E}_{i}\right\}+1 \\
& =f\left(E_{i}\right)+1 .
\end{aligned}
$$

From (i) and (ii), each $E_{i} \in \mathcal{E}$ satisfies $f\left(E_{i}\right)<d\left(E_{i}\right)$, i.e., $E_{i}$ is deficient.

Lemmas 17 and 19 imply the following result, which immediately implies the necessity part of Theorem 4 .

Theorem 20 Let $\mathcal{E} \subseteq 2^{V}$ be a tree Sperner hypergraph. Then there is a weight function $w: \mathcal{E} \rightarrow \mathbb{R}_{+}$such that $\mathcal{E}=\mathcal{W}(f, d)$ holds for two set functions $f$ and $d$ defined as above.

\section{Submodular and Posi-modular Systems}

In this section, we assume that a function $f$ is posi-modular and submodular and a function $d$ is given by one of the following two forms, since applications discussed in the subsequent section satisfy this assumption.

1. For a given function $p: V \rightarrow \mathbb{R}_{+}$, let

$$
d(X)= \begin{cases}\max \{p(v) \mid v \in X\} & \text { if } X \neq \emptyset \\ 0 & \text { if } X=\emptyset .\end{cases}
$$

2. For a given function $r: V^{2} \rightarrow \mathbb{R}_{+}$, let

$$
d(X)= \begin{cases}\max \{r(u, v) \mid u \in X, v \in V-X\} & \text { if } X \neq \emptyset, V \\ 0 & \text { if } X=\emptyset \text { or } V .\end{cases}
$$

It is not difficult to see that $d$ is modulotone in both cases.

Theorem 20 together with the result in $[6,7]$ implies that problem (1) with a posi-modular function $f$ and a modulotone function $d$ is solvable in $\mathrm{O}\left(n^{3} \rho(n)\right)$ time, if the feasibility (i.e., a given $R \subseteq V$ satisfies $f(X) \geq d(X)$ for all $X \subseteq V-R)$ can be checked in $\mathrm{O}(\rho(n))$ time. We first show that the feasibility (transversal) check is possible in polynomial time if $f$ is posimodular and submodular and $d$ is given by either (11) or (12), which implies polynomiality of the problem. We then improve the complexity by using maximal $s$-avoiding $t$-solid sets, which will be defined later, where a similar technique can be found in [2].

We remark that it is open whether the feasibility check is possible in polynomial time for a posi-modular function $f$ and a modulotone function d. 


\subsection{Transversal check}

We consider how to check whether a given set $R \subseteq V$ is a transversal.

Let us first consider a function $d$ of the form (12). In this case, a subset $R \subseteq V$ is a transversal, i.e., $f(X) \geq d(X)$ for each $X \subseteq V-R$ if and only if

$$
\min \{f(X) \mid u \in X \subseteq V-(R \cup\{v\})\} \geq r(u, v)
$$

for each ordered pair $(u, v) \in V^{2}$. The value of the left-hand side of (13) is the minimum value of the submodular function $f^{\prime}: 2^{V-(R \cup\{u, v\})} \rightarrow \mathbb{R}_{+}$ defined by

$$
f^{\prime}(X)=f(X \cup\{u\}) .
$$

Therefore we can check whether $R$ is a transversal by minimizing the submodular function $f^{\prime}$ for every ordered pair $(u, v) \in V^{2}$. Since the submodular function minimization is solved in $\mathrm{O}\left(\left(n^{6} \gamma+n^{7}\right) \log n\right)$ time [9], where $\gamma$ denotes the time required to compute the function value for each subset $X$, problem (1) can be solved in $\mathrm{O}\left(n^{3} \times n^{2} \times \mathrm{O}\left(\left(n^{6} \gamma+n^{7}\right) \log n\right)\right)=$ $\mathrm{O}\left(\left(n^{11} \gamma+n^{12}\right) \log n\right)$ time.

Similarly, for functions $d$ given by (11), the problem can be solved in $\mathrm{O}\left(\left(n^{10} \gamma+n^{11}\right) \log n\right)$ time.

In the subsequent sections, we reduce these complexities.

\subsection{Computing $s$-avoiding solid sets}

For $s, t \in V$ with $s \neq t$, by an $s$-avoiding $t$-solid set $X$ we mean a $t$-solid subset of $V-\{s\}$. An $s$-avoiding $t$-solid set $X$ is called maximal if $X$ is not included in any other $s$-avoiding $t$-solid set. For each $s \in V$, let $\mathcal{S}^{(s)}$ be the family of maximal $s$-avoiding $t$-solid sets for $t \in V-\{s\}$, and let $\mathcal{S}^{*}(f)=\bigcup_{s \in V} \mathcal{S}^{(s)}$.

We consider minimizing a submodular function $f$, in particular, finding a subset $X$ of $V-\{s\}$ containing $t$ such that

$$
f(X)=\min \{f(Y) \mid t \in Y \subseteq V-\{s\}\} .
$$

From the submodularity of $f$, the family of the minimizers is closed under taking union and intersection. Let $N_{t}^{s}$ denote a unique minimal member of this family.

Lemma 21 For $s, t \in V$ with $s \neq t, N_{t}^{s}$ is a unique maximal $s$-avoiding t-solid set.

Proof. From the definition, it is easy to show that $N_{t}^{s}$ is a maximal $s$ avoiding $t$-solid set. Then we only prove the uniqueness. 
By the submodularity of $f$, for any $X \subseteq V-\{s\}$ with $t \in X \nsubseteq N_{t}^{s}$, we have

$$
f(X)+f\left(N_{t}^{s}\right) \geq f\left(X \cap N_{t}^{s}\right)+f\left(X \cup N_{t}^{s}\right) \geq f\left(X \cap N_{t}^{s}\right)+f\left(N_{t}^{s}\right) .
$$

It follows that $f(X) \geq f\left(X \cap N_{t}^{s}\right)$ and $X \cap N_{t}^{s}$ is a proper subset of $X$ containing $t$, which implies that $X$ is not $t$-solid.

Based on this, the family $\mathcal{S}^{(s)}$ can be obtained by computing all sets $N_{t}^{s}$ for $t \in V-\{s\}$. We note that a unique minimal minimizer for a submodular function can be computed by using (strongly) polynomial algorithms for submodular function minimization (e.g., [4, 9, 10, 12]) once. The best known algorithm due to [9] computes a maximal minimizer. Since the minimal minimizer can be obtained by executing it for the submodular function $f^{*}$ defined by $f^{*}(X)=f(V-X)$ for all $X \subseteq V, N_{t}^{s}$ can be computed in $\mathrm{O}\left(\left(n^{6} \gamma+n^{7}\right) \log n\right)$ time [9]. Thus $\mathcal{S}^{*}(f)$ can be computed in $\mathrm{O}\left(\left(n^{8} \gamma+\right.\right.$ $\left.\left.n^{9}\right) \log n\right)$ time.

\subsection{Computing a basic tree for $\mathcal{S}(f)$}

From Theorem 3, given a tree hypergraph with the explicit list of the hyperedges, we can compute a basic tree and the algorithm is polynomial in $n=|V|$ and $m=|\mathcal{E}|[2]$.

Since $\left|\mathcal{S}^{*}(f)\right|$ is at most $n^{2}$ as mentioned above, we can compute a basic tree $T$ for $\mathcal{S}^{*}(f)$ in polynomial time. Moreover, we can show that $T$ is also a basic tree for $\mathcal{S}(f)$, where a similar proof can be found in [2].

Lemma 22 If $T$ is a basic tree for $\mathcal{S}^{*}(f)$, then it is basic for $\mathcal{S}(f)$.

Proof. Let $T$ be a basic tree for $\mathcal{S}^{*}(f)$. Suppose that $T$ is not basic for $\mathcal{S}(f)$, that is, for some $v \in V$ there is a $v$-solid set $X$ that does not induce a subtree of $T$. Then there are two elements $a, b$ of $X$ so that a unique path $P$ in $T$ between $a$ and $b$ contains an element $s \notin X$. This $X$ is an $s$-avoiding $v$-solid set and hence there is a maximal $s$-avoiding $v$-solid set $X^{\prime}$ including $X$. But $T$ is basic for $\mathcal{S}^{*}(f)$ and hence $P$ must belong to $X^{\prime}$, a contradiction.

\subsection{Computing a minimum transversal}

In this section, we consider the problem of computing a minimum transversal $R$ of $\mathcal{W}(f, d)$, i.e., a minimum size set $R \subseteq V$ such that $R \cap X \neq \emptyset$ for all $X \in \mathcal{W}(f, d)$.

Theorem 4 implies that $\mathcal{W}(f, d)$ is a tree hypergraph, and it follows from Lemmas 16 and 22 that a basic tree $T$ for $\mathcal{W}(f, d)$ can be computed in polynomial time. It is known (e.g., [2]) that if a basic tree $T$ is available, 
we can compute a minimum transversal by the following simple algorithm which uses the transversal check as a subroutine.

Choose an arbitrary element $r$ of $T$, and regard $T$ as an arborescence with a root $r$. Here $T[U]$ denotes the subtree of $T$ induced by a vertex set $U$.

\section{Algorithm MinTransversal}

Input: A posi-modular function $f: 2^{V} \rightarrow \mathbb{R}$, a modulotone function $d$ : $2^{V} \rightarrow \mathbb{R}$ with $f(\emptyset) \geq d(\emptyset)$, and a basic tree $T$ for $\mathcal{W}(f, d)$.

Output: A minimum transversal $R$ of $\mathcal{W}(f, d)$.

Step 1. Initialize $R:=\emptyset$ and $U:=V$.

Step 2. If $U$ is empty, then output $R$ and halt.

Step 3. Choose a leaf $v$ of $T[U]$ and $U:=U-\{v\}$.

Step 4. If $R \cup U$ is not a transversal then $R:=R \cup\{v\}$. Go to Step 2 .

Lemma 23 Algorithm MinTransversal outputs a minimum transversal of $\mathcal{W}(f, d)$.

Proof. Let $R^{*}$ be a set which the algorithm outputs. Since $R^{*}$ is clearly a transversal, we only prove the optimality of $R^{*}$. Let $D(v)$ be the set of all descendants of $v$ in $T$ (containing $v$ ). Let $R(v)$ and $U(v)$ respectively denote $R$ and $U$ after Step 3 of the iteration in which $v$ is chosen. Note that for every $v \in V, R(v) \cup U(v) \cup\{v\}$ is a transversal. If $R(v) \cup U(v)$ is not a transversal, then there exists a hyperedge $X_{v}$ with $v \in X \subseteq D(v)-R(v)$. For each $w \in R(v), w \notin X_{v}$ implies that $X_{v} \cap D(w)=\emptyset$, since each hyperedge induces a subtree of $T$. Therefore, we have $X_{v} \cap X_{w}=\emptyset$ for all $v, w \in R^{*}$ with $v \neq w$. This implies that the family $\left\{X_{v} \mid v \in R^{*}\right\}$ is a matching. Since any transversal $R$ satisfies $R \cap X_{v} \neq \emptyset$ for all $X_{v}, v \in R^{*}$, we have $|R| \geq\left|R^{*}\right|$, which completes the proof.

\subsection{Complexity}

Given a posi-modular and submodular function $f: 2^{V} \rightarrow \mathbb{R}_{+}$and a function $d: 2^{V} \rightarrow \mathbb{R}_{+}$given by either (11) or (12), the algorithm outlined above for finding a minimum-size set $R \subseteq V$ such that $f(X) \geq d(X)$ for each $X \subseteq V-R$ consists of the following three steps:

1. Computing the family $\mathcal{S}^{*}(f)$.

2. Computing a basic tree $T$ for $\mathcal{S}^{*}(f)$. 
3. Computing a minimum transversal $R$ of the family $\mathcal{W}(f, d)$ of all minimal deficient sets using $T$ (Algorithm MinTransversal).

As discussed in Section 5.2, Step 1 can be done in $\mathrm{O}\left(\left(n^{8} \gamma+n^{9}\right) \log n\right)$ time. In Step 2, we first determine the weight function $c$ in Theorem 3 and then construct a maximum weight spanning tree $T$. These can be executed in $\mathrm{O}\left(n^{2}\left|\mathcal{S}^{*}(f)\right|\right)=\mathrm{O}\left(n^{4}\right)$ time, since $\left|\mathcal{S}^{*}(f)\right| \leq n^{2}$. Since the time-consuming part of Step 3 (i.e., Algorithm MinTransversal) is to check whether $R \cup U$ is a transversal of $\mathcal{W}(f, d)$ for every $v \in V$, Step 3 can be performed in $\mathrm{O}\left(\left(n^{8} \gamma+n^{9}\right) \log n\right)$ time and $\mathrm{O}\left(\left(n^{9} \gamma+n^{10}\right) \log n\right)$ time for functions $d$ given by (11) and (12), respectively. The time bound of Step 3 dominates the time complexity of the entire algorithm.

Theorem 24 Let $f$ be a posi-modular and submodular function. Then problem (1) can be solved in $\mathrm{O}\left(\left(n^{8} \gamma+n^{9}\right) \log n\right)$ and $\mathrm{O}\left(\left(n^{9} \gamma+n^{10}\right) \log n\right)$ time if $d$ is given by (11) and (12), respectively.

We note that the complexities above are essentially $\mathrm{O}\left(n^{2} S F M(n)\right)$ and $\mathrm{O}\left(n^{3} S F M(n)\right)$, where $S F M(n)$ denotes the time complexity for minimizing a submodular function on $2^{V}$ with $n=|V|$. We also note that the algorithms are quadratically faster than the ones based on $[6,7]$ (See Section $5.1)$.

\section{Applications of Problem (1)}

In this section, we briefly discuss applications of our problem, where we focus on the source location problem and the external network problem in undirected graphs.

Let $G=(V, E)$ be an undirected graph with a capacity function $c$ : $E \rightarrow \mathbb{R}_{+}$. It has a demand function $p: V \rightarrow \mathbb{R}_{+}$. Then the source location problem with edge-connectivity requirements in undirected graphs $[1,8,13$, $14]$ is given by

$$
\begin{array}{ll}
\text { Minimize } & |S| \\
\text { subject to } & \lambda_{G}(S, v) \geq p(v) \text { for all } v \in V \\
& S \subseteq V,
\end{array}
$$

where $\lambda_{G}(S, v)$ denotes the maximum flow value (i.e., edge-connectivity) between $S$ and $v$ in $G$, and we define $\lambda_{G}(S, v)=+\infty$ if $v \in S$. This problem has been studied as a location problem concerned with network reliability.

Suppose that we are asked to locate a set $S$ of multiple servers which can provide a certain service in a multimedia network $\mathcal{N}$. A user at vertex $v$ can receive a service by connecting to a server in $S$ through a path in $\mathcal{N}$. To ensure the quality of the service to $v$ even if certain number $p(v)-1$ of 
links become out of order, we should select $S$ so that the edge-connectivity between $S$ and $v$ is at least $p(v)$. Therefore, this kind of fault-tolerant setting can be formulated as the source location problem.

For each $X \subseteq V$, let $f(X)$ denote the sum of edge capacities between $X$ and $V-X$, i.e.,

$$
f(X)=\sum\{c(u, v) \mid u \in X, v \in V-X,(u, v) \in E\} .
$$

It is well known that $f$ is called a cut function and is posi-modular and submodular. Then by the max-flow min-cut theorem, the source location problem can be regarded as problem (1) when $f$ and $d$ are, respectively, given by (17) and (11).

Let us next consider that the external network problem with edgeconnectivity requirements in undirected graphs [7].

Let $G=(V, E)$ be an undirected graph with a capacity function $c: E \rightarrow$ $\mathbb{R}_{+}$. It has a demand function $r: V^{2} \rightarrow \mathbb{R}_{+}$. Then the external network problem is given by

$$
\begin{array}{ll}
\text { Minimize } & |S| \\
\text { subject to } & \lambda_{G / S}(u, v) \geq r(u, v) \text { for all }(u, v) \in V^{2} \\
& S \subseteq V,
\end{array}
$$

where $G / S$ denotes the graph obtained from $G$ by identifying vertex set $S$ with a single vertex $s$, and if $u \in S$, we define $\lambda_{G / S}(u, v)=\lambda_{G / S}(s, v)$. Similarly to the source location problem, this has been studied as a network reliability problem [7]. Let $f$ and $d$ be respectively given by (17) and (12). Then by the max-flow min-cut theorem, the external network problem can be formulated as problem (1).

Let us now apply the algorithm given in Section 5.5 to these problems. In Step 1, for each $s$ and $t, N_{t}^{s}$ can be computed in $\mathrm{O}\left(n m \log \left(n^{2} / m\right)\right)$ time [5], where $n=|V|$ and $m=|E|$. Thus Step 1 can be done in $\mathrm{O}\left(n^{3} m \log \left(n^{2} / m\right)\right)$ time. As mentioned in Section 5.5, Step 2 can be done in $\mathrm{O}\left(n^{4}\right)$ time. Since each transversal check in Step 3 is, respectively, possible in $\mathrm{O}\left(n^{2} m \log \left(n^{2} / m\right)\right)$ and $\mathrm{O}\left(n^{3} m \log \left(n^{2} / m\right)\right)$ time for $d$ given by (11) and (12), Step 3 can be done in $\mathrm{O}\left(n^{3} m \log \left(n^{2} / m\right)\right)$ and $\mathrm{O}\left(n^{4} m \log \left(n^{2} / m\right)\right)$ time, respectively.

Therefore, by using our general framework given in Section 5.5, we have the following result.

Corollary 25 The source location problem and the external network problem are solvable in $\mathrm{O}\left(n^{3} m \log \left(n^{2} / m\right)\right)$ and $\mathrm{O}\left(n^{4} m \log \left(n^{2} / m\right)\right)$ time, respectively.

We remark that this is the first polynomial-time algorithm for the external network problem, and it is known that the source location problem can 
be solved in $\mathrm{O}\left(n^{2} m \log \left(n^{2} / m\right)\right)$ time by using its own specific properties [1]. We also note that our general framework is applicable for the source location problem and the external network problem for not only graphs $G=(V, E)$ but also hypergraphs $(V, \mathcal{E})$, where $f$ is given by

$$
f(X)=\sum\{c(E) \mid E \cap X, E \cap(V-X) \neq \emptyset, E \in \mathcal{E}\} .
$$

\section{Concluding Remarks}

In this paper we considered the problem of finding a minimum transversal of the hypergraph of all minimal deficient sets of a given system $(V, f, d)$. We analyzed the hypergraph of minimal deficient sets of a posi-modular system and proved that it is a tree hypergraph. Then we described a polynomial-time algorithm if $f$ is also submodular and $d$ is defined by $d(X)=\max \{r(v, w) \mid v \in X, w \in V-X\}$ for a function $r: V^{2} \rightarrow \mathbb{R}_{+}$. Moreover, we showed that a tree hypergraph consisting of a Sperner family can be described by minimal deficient sets of a posi-modular system with a modulotone function. That is, we provided a new characterization of tree hypergraphs.

Some problems remain for further work. One issue is to construct a polynomial-time algorithm when the demand function is a general modulotone function, while the algorithm in this paper only addresses a function by (12) or (11). Another issue is to construct a polynomial-time algorithm for posi-modular function minimization or to prove that it is NP-hard. As mentioned in Section 4, Theorem 4 and the good properties of solid sets follow from the posi-modularity, and the submodularity is required only from the algorithmic aspect. Therefore if we have a polynomial-time algorithm for posi-modular function minimization, then the argument in this paper is completed by the posi-modularity.

Acknowledgements This research was partially supported by the Scientific Grant-in-Aid from Ministry of Education, Science, Sports and Culture of Japan.

\section{References}

[1] K. Arata, S. Iwata, K. Makino, and S. Fujishige: Locating sources to meet flow demands in undirected networks, J. Algorithms, 42 (2002), $54-68$.

[2] M. Bárász, J. Becker, and A. Frank: An algorithm for source location in directed graphs, Operations Research Letters, 33 (2005), 221-230. 
[3] A. Brandstädt, V. B. Le, and J. P. Spinrad: Graph Classes: A Survey, SIAM Monographs on Discrete Mathematics and Applications, SIAM, Philadelphia (1999).

[4] S. Fujishige: Submodular Functions And Optimization: Second Edition, Annals of Discrete Mathematics, 58, Elsevier (2005).

[5] J. Hao and J. B. Orlin: A faster algorithm for finding the minimum cut in a graph J. Algorithms, 17 (1994), 424-446.

[6] J. van den Heuvel and M. Johnson: Transversals of subtree hypergraphs and the source location problem in digraphs, CDAM Research Report, LSE-CDAM-2004-10, London School of Economics.

[7] J. van den Heuvel and M. Johnson: The external network problem with edge- or arc-connectivity requirements, Workshop on Combinatorial and Algorithmic Aspects of Networking, CAAN 2004, Lecture Notes in Computer Science 3405, Springer (2004), 114-126.

[8] H. Ito, H. Uehara, and M. Yokoyama: A faster and flexible algorithm for a location problem on undirected flow networks, IEICE Trans., E83-A (2000), 704-712.

[9] S. Iwata: A faster scaling algorithm for minimizing submodular functions, SIAM J. Comput., 32 (2003), 833-840.

[10] S. Iwata, L. Fleischer, and S. Fujishige: A combinatorial strongly polynomial algorithm for minimizing submodular functions, J. of $A C M, 48$ (2001), 761-777.

[11] T. A. Mckee and F. R. McMorris: Topics in Intersection Graph Theory, SIAM Monographs on Discrete Mathematics and Applications, SIAM, Philadelphia (1999).

[12] A. Schrijver: A combinatorial algorithm minimizing submodular functions in strongly polynomial time, Journal of Combinatorial Theory, Series B, 80 (2000), 346-355.

[13] H. Tamura, M. Sengoku, S. Shinoda, and T. Abe: Some covering problems in location theory on flow networks, IEICE Trans., E75-A (1992), $678-683$.

[14] H. Tamura, H. Sugawara, M. Sengoku, and S. Shinoda: Plural cover problem on undirected flow networks, IEICE Trans., J81-A (1998), 863869 (in Japanese). 